\title{
Magnetic resonance imaging of sea-ice pore fluids: methods and thermal evolution of pore microstructure
}

\author{
H. Eicken ${ }^{\mathrm{a}, *}$, C. Bock ${ }^{\mathrm{b}}$, R. Wittig ${ }^{\mathrm{b}}$, H. Miller ${ }^{\mathrm{b}}$, H.-O. Poertner ${ }^{\mathrm{b}}$ \\ ${ }^{a}$ Geophysical Institute, University of Alaska Fairbanks, P.O. Box 757320, Fairbanks, AK 99775-7320, USA \\ b Alfred Wegener Institute for Polar and Marine Research, P.O. Box 120161, D-27515 Bremerhaven, Germany
}

Received 29 October 1999; accepted 8 July 2000

\begin{abstract}
Microstructure and thermal evolution of sea-ice brine inclusions were investigated with magnetic resonance imaging (MRI) techniques. Ice samples were kept at temperatures between $-2{ }^{\circ} \mathrm{C}$ and $-25^{\circ} \mathrm{C}$ during ${ }^{1} \mathrm{H}$ imaging in a $4.7-\mathrm{T}$ magnet at $200 \mathrm{MHz}$. Measurements were completed in a 20-cm diameter cylindrical probe and actively shielded gradient coils (max. $50 \mathrm{mT} \mathrm{m}^{-1}$, pixel dimensions $>0.2 \mathrm{~mm}$, slice thicknesses $>1 \mathrm{~mm}$ ), and for higher resolution in a mini-imaging unit with a 9-cm diameter probe with gradient coils of $200 \mathrm{mT} \mathrm{m}^{-1}$ (pixel dimensions $<0.1 \mathrm{~mm}$, slice thickness $<0.4 \mathrm{~mm}$ ). Absorption of radio-frequency (RF) signals in the dielectrically lossy brine resulted in degraded signals and was alleviated by use of a contrast agent (decane). MRI data and sea-ice thin section images agree very well $(<5 \%$ deviation for pore microstructural parameters). Analysis of ice grown under different current speeds indicates that pores are smaller and pore number densities larger at higher current speeds. The thermal evolution of fluid inclusions was studied on cold first-year ice samples, maintained at close to in-situ temperatures prior to experiments. Warming from $-21^{\circ} \mathrm{C}$ to $-10^{\circ} \mathrm{C}$ to $-6^{\circ} \mathrm{C}$ is associated with a distinct increase in pore size (from 1.5 to 1.7 to $2.6 \mathrm{~mm}$ for the upper 10-percentile in the vertical) and elongation (4.0 to 4.2 to 6.2 for ratio of major to minor pore axes in the vertical) and a decrease in number densities $(0.75$ to 0.62 to $0.58 \mathrm{~mm}^{-3}$ in the vertical). Aspect ratios increased from 4:2:1 to 6:2:1 (upper 10-percentile), indicating expansion and merging of pores in the vertical, possibly promoted by microscopic residual brine inclusions. (C) 2000 Elsevier Science B.V. All rights reserved.
\end{abstract}

Keywords: Sea ice; Nuclear magnetic resonance; Pore structure; Crystal growth; Chukchi Sea; Image analysis

\section{Introduction}

Sea ice is distinct from other types of ice present at the earth's surface owing to the ubiquitous occurrence of sub-millimeter to decimeter-sized inclusions

\footnotetext{
* Corresponding author. Fax: +1-907-474-7290.

E-mail address: hajo.eicken@gi.alaska.edu (H. Eicken).
}

of liquid brine (Weeks and Ackley, 1986; Weeks, 1998; Wettlaufer, 1998). These inclusions strongly affect radiative and heat transfer through the ice (Perovich, 1998; Weeks, 1998) and they control the electromagnetic properties of sea ice (Hallikainen and Winebrenner, 1992; Golden et al., 1998a,b). As the brine seeks to maintain thermodynamic equilibrium with the ice matrix, the morphology and size of the inclusions depends strongly on the salinity and 
temperature of the ice (Weeks and Ackley, 1986; Perovich and Gow, 1996). Thus, as an ice volume with a salinity of $5 \%$ is cooled from $-2^{\circ} \mathrm{C}$ to $-20^{\circ} \mathrm{C}$, its brine porosity decreases from $122 \%$ to $17 \%$, with part of the brine expelled due to the differential increase in ice over brine volume (Cox and Weeks, 1983). The changes in the pore microstructure associated with spatial and temporal variations in ice temperature are responsible for the distinct seasonal contrast as well as the steep gradients in ice properties observed as a function of depth or ice thickness.

This dependence of pore morphology on ice temperature has proved a significant challenge to the study of the microstructural evolution of sea ice pores. While such studies are prerequisite to improving our understanding of the geophysics of sea ice and its representation in numerical models, standard methods of sample processing and thin-section analysis are not necessarily suited for the quantitative analysis of three-dimensional pore structure as a function of thermal forcing. First, to avoid loss of brine and preserve ice microstructure, storage and processing of ice samples commonly occurs at very low temperatures, ideally below the precipitation point of hydrohalite at $-22.8^{\circ} \mathrm{C}$ (see, e.g., recommendations for standardized ice processing and sample analysis techniques in Schwarz et al., 1981, or discussion by Eicken et al., in preparation). This results in shrinkage of pores and to some extent in loss of brine from the sample (Cox and Weeks, 1986). At higher temperatures (above $-5^{\circ} \mathrm{C}$ ) closer to typical in-situ temperatures, loss of brine is even more a concern due to the high permeability of the samples. The second problem is of a stereological nature, insofar as optical thin-section analysis generally deals with samples that are too thin to allow for full reconstruction of the three-dimensional pore space. In analysing such conventionally recorded thin-section images, samples are then mostly assumed to be of zero thickness with important stereological implications of an entirely different nature (Eicken, 1993; Weeks, 1998).

As has been pointed out both in the context of glaciology (Kawamura, 1988; Edelstein and Schulson, 1991) as well as in other fields of petrophysics (e.g., Lindquist et al., 1996), non-destructive, tomographic methods of structural analysis provide for a potentially elegant solution of this dilemma. Thus, Kawamura (1988, 1990) employed X-ray computed tomography (CT) which relies on the strong contrast in the X-ray absorption coefficients for ice, air and brine to derive the morphology of macro-pores and the density structure of sea ice and firn cores. Further work along these lines has been carried out by Lange (1988) and Hannke (1994). Edelstein and Schulson (1991) took a different approach in applying nuclear magnetic resonance imaging (MRI) techniques to the study of $\mathrm{NaCl}$ ice, discriminating between the relaxation of liquid- and solid-phase hydrogen atoms precessing in an externally imposed magnetic field. All these investigations focussed on pores of millimetersize or larger owing to limitations in the resolution provided by the instrument and technique (with pixel sizes $>1 \mathrm{~mm}$ and slice thicknesses between 2 and $10 \mathrm{~mm}$ for CT and $0.5 \mathrm{~mm}$ with slice thickness 5 $\mathrm{mm}$ for MRI). While the CT studies were all performed at an arbitrary, variable temperature lower than the original in-situ temperature, Edelstein and Schulson provided a qualitative comparison between the microstructure of doped $\mathrm{NaCl}$ ice at $-4^{\circ} \mathrm{C}$ and $-11^{\circ} \mathrm{C}$. A novel, entirely different approach to overcome the problems related to sample extraction has been taken by Callaghan et al. (1999). Utilizing the earth's magnetic field to observe nuclear precession, Callaghan et al. were able to determine the brine volume fraction directly at the site or even in situ and furthermore extended the method to measurements of brine diffusivities. However, this approach did not allow for discrimination between different pore sizes or populations based on imaging methods.

Here, we present data on sea-ice pore microstructure based on MRI in a 4.7-T magnet with actively shielded gradient coils (50 and $200 \mathrm{mT} \mathrm{m}^{-1}$ ) under variable thermal conditions. The major aims of this work are to (1) increase image resolution to values comparable to standard thin-section analysis $(0.1 \mathrm{~mm}$ with slice thickness less than $0.5 \mathrm{~mm}$ ), (2) develop discrimination and segmentation techniques in order to extract quantitative microstructural data from MRI data sets, including the use of contrast agents to alleviate some of the problems inherent in transmission of radio-frequency (RF) resonator signals, and (3) derive a first data set on the three-dimensional evolution of pore microstructure under variable thermal forcing based on the analysis of ice samples that 
have been maintained at the in-situ temperature after sampling. The ice samples originate both from icetank experiments devoted to the study of microstructural evolution (Eicken et al., 1998) as well as from coastal sea ice obtained at Barrow in northern Alaska. Apart from a discussion of methodological aspects, the evolution of pore microstructure will receive particular attention in the context of ice permeability and fluid transport at lower temperatures (Golden et al., 1998a,b; Freitag, 1999), as the present contribution is part of a study devoted to fluid transport and its importance for survival of microorganisms within sea-ice pores at low temperatures (Eicken et al., 1999; Deming and Huston, in press; Junge et al., in press).

\section{Methods}

\subsection{Principles and instrumentation of MRI}

The detection of water within a multi-phase system with nuclear magnetic resonance (NMR) techniques is a well-established technique and among other topics has also been applied to problems in sea-ice research to study the thermal evolution of the brine fraction in sea ice (Richardson and Keller, 1966; Richardson, 1976; Melnichenko et al., 1979). MRI is based on the principle that the resonance frequency depends on the position of a nucleus in a magnetic field gradient (Wehrli, 1988; Callaghan, 1991). The frequency spectrum obtained over a sample volume can then be decomposed into individual frequencies (containing information about the $x-y$ position) and amplitudes (containing information about the spin, or in the case of ${ }^{1} \mathrm{H}$, proton density). Image acquisition and in particular the spatial resolution are determined by the timing and characteristics of the RF pulses as well as the magnitude of the field gradient across the sample volume.

The present study has been carried out in a Bruker Biospec 47/40 imaging spectrometer (Bruker, Ettlingen, Germany), at 4.7 $\mathrm{T}$ (Larmor frequency for ${ }^{1} \mathrm{H}=200 \mathrm{MHz}$ ) equipped with actively shielded gradient coils providing field gradients of $50 \mathrm{mT} \mathrm{m}^{-1}$ and a mini-imaging unit with gradient field strength of up to $200 \mathrm{mT} \mathrm{m}^{-1}$. Cylindrical probes adapted to high-conductivity samples were used for RF signal transmission and detection (20- and 9-cm diameter, respectively). Image acquisition was mostly confined to a spin-echo method (multi-slice multi-echo, MSME, matrix size up to $1024 \times 512$, field-of-view (FOV) up to $12 \times 12 \mathrm{~cm}$ (standard imaging, S) or $6 \times 6 \mathrm{~cm}$ (mini imaging, M), slice thickness down to $1.2 \mathrm{~mm}(\mathrm{~S})$ or $0.3 \mathrm{~mm}(\mathrm{M})$, slice separation down to $5 \mathrm{~mm}(\mathrm{~S})$ or $2 \mathrm{~mm}(\mathrm{M})$, with sinc3 RF pulses of 3000- $\mu$ s duration) or a gradient-echo method (FLASH, flip angle $22.5^{\circ}$, sinc3 of $3000 \mu \mathrm{s}$ ) with typical echo times TE of $17.5 \mathrm{~ms}$ for MSME and 5 $\mathrm{ms}$ for FLASH. Repetition time TR was on the order of 300-2000 ms for MSME and 400-500 ms for FLASH. Typically, measurements for individual samples lasted between 4 and $12 \mathrm{~h}$, averaging over up to eight samples in order to increase the signalto-noise ratio (SNR).

\subsection{Samples and sample preparation}

In order to optimize MRI system performance and to develop and improve upon methods of MRI data analysis, a first set of measurements was carried out on artificial sea ice grown under fully controlled environmental conditions. These samples originated from ice-growth experiments in the Environmental Arctic Test Basin (EATB) of the Hamburgische Schiffbauversuchsanstalt in 1996 and 1997 (INTERICE I, Eicken et al., 1998). In a basin of dimensions $30 \times 6 \times 1.2 \mathrm{~m}$, a congelation-type sea-ice sheet was grown from artificial seawater, prepared by dissolving Aquarium Systems "Instant Ocean" artificial seasalt in tapwater, to between 0.15- and 0.2-m thickness (for details, see Eicken et al., 1998). The resulting ice is representative of new ice grown under natural conditions and exhibits the same inventory of small- and large-scale pore features (Cottier et al., 1999; Eicken et al., in preparation).

Samples for the studies of sea-ice thermal evolution were obtained in March 1999 from the fast-ice cover near Barrow (sampling location at $71^{\circ} 19.95^{\prime} \mathrm{N}$ $156^{\circ} 40.55^{\prime} \mathrm{W}$ ), on the northern coast of Alaska. Numerous studies of the ice cover at this location have shown it to be representative of homogeneous firstyear, undeformed Arctic congelation-type sea ice (Weeks and Gow, 1978; Cole and Shapiro, 1998). At 
the time of sampling, ice thickness was approximately $1.15 \mathrm{~m}$, with a snow cover of $0.06-\mathrm{m}$ depth.

Standard storage, processing and analysis of seaice samples generally occurs at ambient temperatures below $-20^{\circ} \mathrm{C}$ to minimize loss of brine and sample degradation (Schwarz et al., 1981; Lange, 1988). Furthermore, samples are often subjected to significant temperature variations during transport and storage which are associated with significant changes in fractional brine volume (Cox and Weeks, 1986) and possibly changes in microstructure. Two different approaches have been taken to minimize or exclude artifacts as a result of thermal changes. For the INTERICE samples, brine was removed from the ice matrix by centrifuging the samples at approximately $70 \times g$ under in-situ temperatures in a Beckman GS6 refrigerated centrifuge (Eicken et al., in preparation). This method has been shown to remove about $80 \%$ of the brine content at temperatures typical of the experiments (Weissenberger et al., 1992). It leaves the pore space filled with air rather than brine and hence unaffected by changes in temperature. After centrifugation, samples were stored and processed at $-25^{\circ} \mathrm{C}$ to $-30^{\circ} \mathrm{C}$. Prior to MRI data acquisition, samples were slowly warmed to the in-situ temperature and were immersed in either artificial brine (at a concentration in equilibrium with the ice at in-situ temperature) or $n$-decane as a contrast agent. Gas remaining in smaller brine pockets was driven from the sample by centrifugation at in-situ temperature at less than $50 \times g$.

The samples collected in Barrow were obtained during the coldest week of the year, with air temperatures mostly between $-30^{\circ} \mathrm{C}$ and $-40^{\circ} \mathrm{C}$ and temperatures in the uppermost ice layers ranging between $-15^{\circ} \mathrm{C}$ and $-20^{\circ} \mathrm{C}$. After extraction of 10-cm-diameter ice cores with a CRREL-type fiberglass-barrel auger, samples were maintained at close to in-situ temperature during storage and transport (mostly within 2-3 K and a maximum deviation of 5 $\mathrm{K}$, with temperatures monitored through "Hobo" temperature loggers manufactured by Onset Computer). Part of the samples were cooled to and stored at $-25^{\circ} \mathrm{C}$ in Fairbanks. Samples were transferred to Bremerhaven for MRI in Dewar vessels and insulated ice-core transport containers with sample temperatures maintained at between $-22^{\circ} \mathrm{C}$ and $-26^{\circ} \mathrm{C}$. Processing of image data was carried out using the

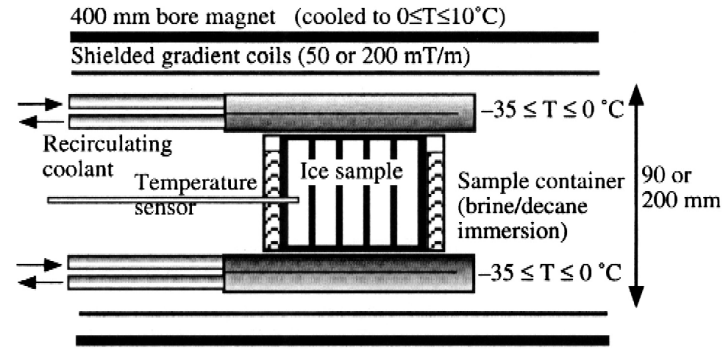

Fig. 1. Transverse cross-section through $400 \mathrm{~mm}$ bore magnet (cooled to between $0^{\circ} \mathrm{C}$ and $10^{\circ} \mathrm{C}$ ), gradient coils, cooling system (with upper and lower cooler plate maintained at $-35^{\circ} \mathrm{C}$ to $0^{\circ} \mathrm{C}$ ) and sample container.

public-domain NIH Image program (written by Wayne Rasband at the U.S. National Institutes of Health and available at http://rsb.info.nih.gov/ nih-image; Rasband and Bright, 1995).

\subsection{Sample temperature control}

In order to maintain the ice at a constant temperature during MRI data acquisition and to be able to impose vertical temperature gradients, samples were kept in a cooled polycarbonate enclosure during the measurements (Fig. 1). For both the smaller-diameter high-resolution imaging unit and the standard system, a synthetic coolant (SYNTH60) circulated through the entire volume of a cooler plate in direct contact with the sample container from above and below. The sample resided in a polycarbonate container, either completely filled with ice or in a liquid immersion. Apart from measuring and controlling the temperature of the recirculating coolant, the sample temperature was determined with a fiber-optic probe (Luxtron 504, Polytec, Waldheim, Germany) in a small-diameter hole drilled into the sample surface. During normal operation, temperatures did not deviate by more than $\pm 1 \mathrm{~K}$ from the setpoint.

\section{Results: methodological aspects}

3.1. MRI of sea-ice pore fluids: constraints on detectability and resolution, injection of a contrast agent

MRI of pore fluids is constrained by a number of factors relating both to the instrumentation and the 
physical properties and microstructure of the sample. Given the lack of sea-ice MRI work, a first step in the present study consisted of an optimization of the instrumental parameters. Analysis of ice samples immersed in brine at the respective in-situ temperatures established that the SNR of gradient-echo FLASH images was poor, most likely due to RF loss in the brine, and that the images showed artifacts due to susceptibility changes within the ice matrix (resulting from air bubbles, brine-ice transitions, eddy currents). Spin-echo images yielded results that were not severely compromised by artifacts due to eddy currents induced in the brine layer surrounding the sample (5- to 10-mm thick, see bottom of Fig. 2).

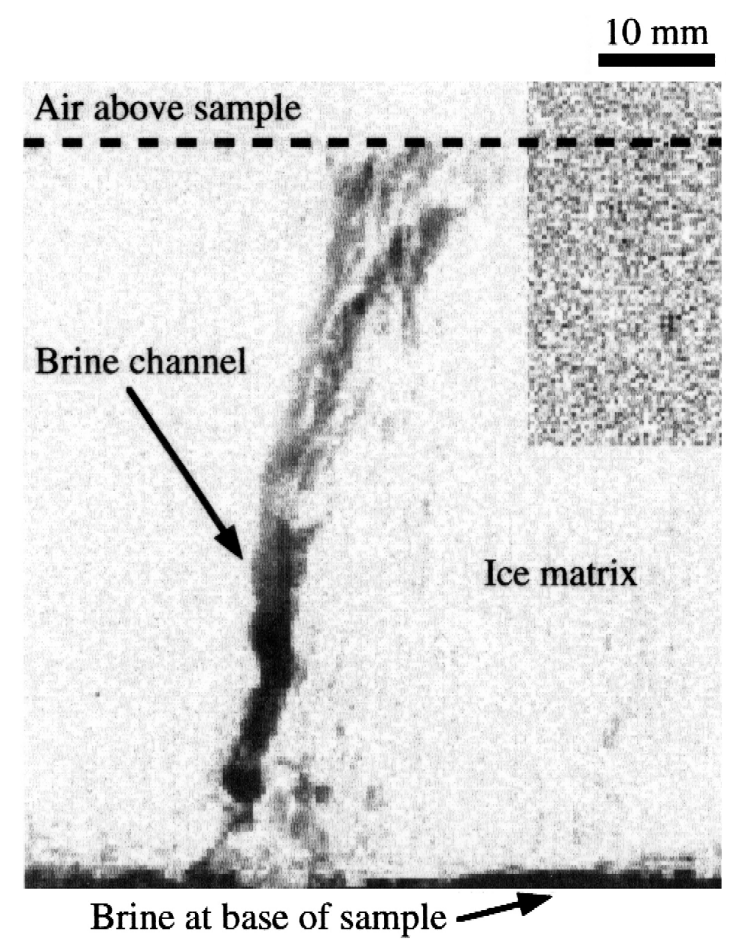

Fig. 2. MRI cross-section through layer of artificial sea ice (sample H961219Q, 0.07- to 0.14-m depth, slice thickness 1.5 $\mathrm{mm}$, pixel size $0.4 \times 0.4 \mathrm{~mm}$ ) recorded in immersion of artificial brine at $-2^{\circ} \mathrm{C}$ with a spin-echo method (MSME). Note that here and in all subsequent images, MRI and thin-section scenes are shown as inverted contrast images (pores appearing dark). While the brine channel in the center is well discernible, very few pore features can be identified in the ice matrix, as is evident from the subregion in the upper right that has been subjected to a linear histogram transform to enhance the contrast in the ice matrix.
However, due to the higher RF power input $\left(90^{\circ}-\right.$ $180^{\circ}$ flip angles) in comparison to FLASH, the probe was heating up at in-situ temperatures, compromising long-term image sampling. As apparent from the vertical cross-section through a new-ice cover shown in Fig. 2, the structure of brine channels and other larger pore features is well-resolved. This includes the upper layers of the sample where the channel is widened and to some extent branched out. As shown by the contrast-enhanced inset in Fig. 2, there is no discernible difference in the signal between ice matrix and overlying air. At a pixel size of approximately $0.4 \mathrm{~mm}$ and a slice thickness of $1.5 \mathrm{~mm}$, smaller brine inclusions are not distinguishable within the ice matrix.

While the resolution of the imaging system as defined by the size of the volume elements (voxels) may not allow for direct discrimination of small-scale pores, it can still be feasible to derive the fraction of such fluid inclusions based on the signal strength integrated over a larger sample sub-volume. Based on Callaghan (1991), the SNR for a signal induced in the receiver coils of radius $r$ during resonance at frequency $f_{0}$ and for a frequency bandwidth $\Delta f$ is directly proportional to the volume fraction $N_{\mathrm{p}}$ of protons (in this case ${ }^{1} \mathrm{H}$ in the brine) in a subvolume $V_{\text {s }}$ such that

$\mathrm{SNR} \approx K \frac{N_{\mathrm{p}} V_{\mathrm{s}} f_{0}^{7 / 4}}{r \Delta f^{1 / 2}}$

with constant $K=1.2 \times 10^{-3} \mathrm{~s}^{5 / 4} \mathrm{~m}^{-2}$ based on instrument parameters and assuming solenoidal coils at room temperature. For a voxel volume of (0.4 $\mathrm{mm})^{3}$ and parameters characteristic of the Bruker system employed in this study, SNR is roughly 100 in order of magnitude, with values decreasing to below 10 as higher resolutions are approached. In solids, the signal is broadened over a wider resonance frequency band. In combination with shorter relaxation times this allows for a direct discrimination between brine and ice, with the signal for pure ice and air being close to indistinguishable (Fig. 2, Table 1). Disregarding the submicroscopic effects on the relaxation of ${ }^{1} \mathrm{H}$ along phase boundaries, the liquid fraction $f_{\mathrm{s}}$ in a given sample volume with a signal of magnitude $S$ can be derived through a set 
Table 1

Magnitude of MRI signal (as digital number DN) and liquid volume fraction $f_{1}$ for phase components and bulk samples immersed in brine

\begin{tabular}{|c|c|c|c|c|}
\hline \multirow[t]{2}{*}{ Sample } & \multirow[t]{2}{*}{ Phase } & \multicolumn{2}{|l|}{$\mathrm{DN}$} & \multirow[t]{2}{*}{$f_{1}(\% o)$} \\
\hline & & Mean & $\sigma$ & \\
\hline E.1.5.8 & air & 8.3 & 1.6 & 0 \\
\hline \multirow[t]{3}{*}{$\left(T=-4^{\circ} \mathrm{C}\right)$} & ice matrix & 9.8 & 2.0 & 17 \\
\hline & brine & 94.5 & 13.5 & 1000 \\
\hline & bulk ice sample & 16.6 & 13.8 & 96 \\
\hline E.2.12.5 & air & 9.6 & 1.8 & 0 \\
\hline \multirow[t]{3}{*}{$\left(T=-4^{\circ} \mathrm{C}\right)$} & ice matrix & 10.8 & 2.1 & 5 \\
\hline & brine & 147.6 & 22.9 & 1000 \\
\hline & bulk ice sample & 11.9 & 6.9 & 10 \\
\hline E.2.9.3 & air & 8.9 & 1.7 & 0 \\
\hline \multirow[t]{3}{*}{$\left(T=-4^{\circ} \mathrm{C}\right)$} & ice matrix & 9.9 & 2.0 & 8 \\
\hline & brine & 125.9 & 13.1 & 1000 \\
\hline & bulk ice sample & 12.5 & 10.5 & 30 \\
\hline E.970819.J11 & air & 7.3 & 1.7 & 0 \\
\hline \multirow[t]{3}{*}{$\left(T=-7^{\circ} \mathrm{C}\right)$} & ice matrix & 8.7 & 2.4 & 10 \\
\hline & brine & 131.5 & 16.9 & 1000 \\
\hline & bulk ice sample & 25.1 & 33.7 & 140 \\
\hline
\end{tabular}

of calibration measurements to determine the signal for pure liquid $S_{1}$ and pure solid $S_{\mathrm{s}}$ according to

$f_{\mathrm{s}}=\frac{S-S_{1}}{S_{\mathrm{s}}-S_{1}}$

Data for several brine-immersion samples are listed in Table 1. The differences apparent between air and brine are a result of the signal generated by brine inclusions that are not resolved in the image data but nevertheless induce a resonator response. While the brine porosity of the ice matrix is derived as between $5 \%$ and $17 \%$ from the MRI data, direct measurements and analysis of thin sections indicate somewhat larger values (Eicken et al., 1998, in preparation). However, the bulk liquid volume fractions integrated over the entire sample volume correspond more closely to values derived from salinity and temperature measurements (Table 1).

These deviations are likely the result of one or more of the following causes. First, short- and longrange interaction between water molecules and the ice surface in the vicinity of phase boundaries are known to affect the relaxation times and detectability of brine (Callaghan, 1991). While we are currently lacking systematic studies of this aspect, theoretical considerations on the characteristic length scales of dispersive molecular forces indicate that this should mostly affect pores in the sub-micrometer size range. Second, the resolution or voxel size has a strong impact on the magnitude of the SNR. The SNR depends on the FOV (as defined by the product of its linear dimensions) as well as the sampling rate of the signal in the frequency domain, defined by the number of samples $n_{\mathrm{p}}$ and $n_{\mathrm{f}}$ encoding phase and frequency of the signal (Wehrli, 1988). As the SNR is proportional to FOV $\times\left(n_{\mathrm{p}} n_{\mathrm{f}}\right)^{-1 / 2}$, the detection of liquid brine at higher resolutions is increasingly limited.

A third aspect is of particular importance for the study of sea ice: transmission of the RF signal and hence, the sensitivity of the probe may be compromised by electromagnetically lossy brine enveloping the sample. The MRI spectrometer operates at 200 $\mathrm{MHz}$ and is equipped with probes for high-conductivity samples such as seawater. Salinities of sea-ice brines typically range between $40 \%$ and $200 \%$, therefore the matching of the probes was not optimal, resulting in higher reflection rates inside the probes. Furthermore, at frequencies below $1 \mathrm{GHz}$, the dielectric loss factor $\varepsilon^{\prime \prime}$ (i.e., the imaginary part of the complex permittivity) for freshwater tends towards zero, whereas that of brine increases, with $\varepsilon^{\prime \prime}$ approximately 500 at $200 \mathrm{MHz}$ (Kovacs et al., 1987). Loss factors for sea ice also increase with decreasing frequencies in this range (Takashima et al., 1992). Such high values of $\varepsilon^{\prime \prime}$ not only dampen the signal significantly, but with a transmitter power of approximately $1 \mathrm{~kW}$ they can also result in significant warming of the sample and the surrounding brine. Whereas studies of the macroscopic pore structure are not necessarily compromised by these constraints, we took two approaches to minimize or remove these problems. For the study of thermally driven changes in pore structure, samples were cut and microtomed to assure a tight fit into the sample container, eliminating the need for a brine immersion.

In a different approach, brine was removed from the ice through centrifuging and the samples were immersed in a liquid contrast agent with more suitable dielectric and thermodynamic properties. Decane $\left(\mathrm{CH}_{3}\left(\mathrm{CH}_{2}\right)_{8} \mathrm{CH}_{3}\right)$ proved to be most useful due to its low freezing point, non-polarity and high pro- 


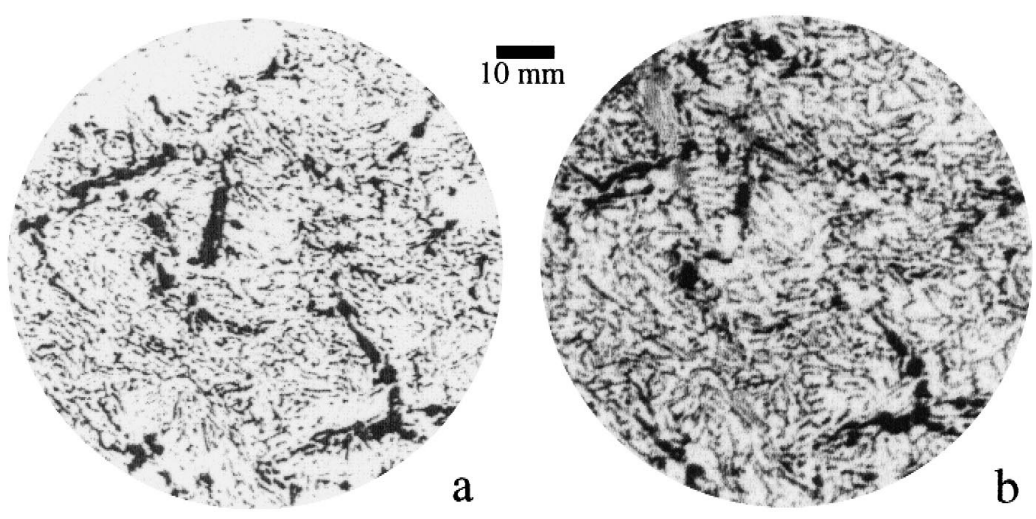

Fig. 3. Comparison of pore microstructure between horizontal thin-section image (a) and MRI data (b) of the same sea-ice sample (H961219Q, 0.05-m depth). Pores appear dark in both images, with both larger secondary brine channels and pockets as well as smaller brine layers discernible. Pore-free areas along margin of upper half of thin-section sample are artifacts due to sample processing (see Table 2 for further details on image acquisition and data).

ton density as well as its immiscibility with water. In this way, spin-echo imaging could be performed with greater matrix sizes to increase resolution, more adequate and shorter pulses resulting in thinner slice thicknesses, and a larger number of signal averages to increase SNR. At the same time, RF heating was significantly reduced due to better probe matching and reduction in eddy currents, allowing for longer measurement periods. Fig. 3 demonstrates that samples saturated with decane resulted in a significant improvement of image quality. Moreover, increased signal strength allowed for a reduction in the linear voxel dimensions by more than half (see instrument parameters shown in Table 2). Thus, in a standard mode of operation with a field gradient of $50 \mathrm{mT}$

Table 2

Comparison between thin-section image and MRI data (sample H961219Q, 0.05-m depth, low-resolution standard gradient coils, see Fig. 3)

\begin{tabular}{llll}
\hline Parameter & $\begin{array}{l}\text { Thin section } \\
\text { data }\end{array}$ & MRI data \\
\cline { 2 - 2 } & Mean $\pm \sigma$ & & Mean $\pm \sigma$ \\
\hline Pixel size (horizontal), mm & 0.18 & & 0.21 \\
Slice thickness (vertical), mm & $<0.05$ & & 1.26 \\
Porosity & 0.187 & & 0.176 \\
Pore area, mm ${ }^{2}$ & $0.76 \pm 3.40$ & & $1.10 \pm 4.40$ \\
Pore perimeter, mm & $3.34 \pm 7.40$ & & $3.97 \pm 8.60$ \\
Pore major axis, $\mathrm{mm}$ & $1.06 \pm 1.23$ & & $1.19 \pm 1.44$ \\
Pore minor axis, $\mathrm{mm}$ & $0.45 \pm 0.44$ & $0.58 \pm 0.56$ \\
\hline
\end{tabular}

$\mathrm{m}^{-1}$ in the 20-cm-diameter resonator, the method appears to sufficiently resolve both larger-scale pores such as brine channels as well as the sub-millimeter brine layers characteristic of columnar sea ice (Fig. 3).

\subsection{Segmentation of MRI data}

Quantitative microstructural analysis requires the segmentation of MRI data into solid ice and fluid inclusions. In the case of MRI this can be approached in a rigorous fashion, given that the signal is proportional to the total number of liquid-phase protons in a given sub-volume. Hence, the signal associated with the pure end-member components $S_{\text {s }}$ and $S_{1}$ (ice and brine or decane) can be employed to constrain Eq. (2) and allow for the derivation of the liquid volume fraction (i.e., porosity) of individual volume elements. $S_{\mathrm{s}}$ and $S_{1}$ can be derived either manually through measurement over a test volume or from a sample histogram such as the one shown in Fig. 4. With the liquid volume fraction computed from the signal of each individual voxel according to Eq. (2), the segmentation criterion can be based on a simple fractional-volume threshold, with all voxels corresponding to a liquid volume fraction $\mathrm{f}_{1} \leq 0.5$ classified as ice and all those with $f_{1}>0.5$ as pores. The classification error due to a finite SNR can then be derived from the FOV and the number of samples averaged for each data set (see Section 5.1). 


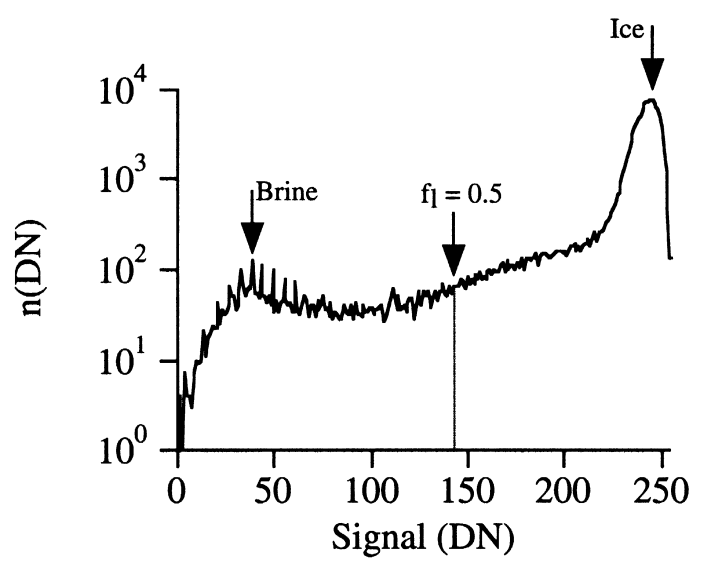

Fig. 4. Histogram of MRI signal for image shown in Fig. 2 (sample H961219Q, 0.07- to 0.14-m depth). Maxima associated with pure brine and ice as well as the segmentation threshold corresponding to a liquid volume fraction $f_{1}=0.5$ are shown.

\subsection{Comparison between ordinary thin sections and MRI data}

While a first, subjective comparison between the thin-section image and the MRI data in Fig. 13 indicates good agreement between the two data sets, a quantitative assessment based on pore microstructural data requires segmentation and derivation of pore parameters from binarized images as outlined in Eicken et al. (in preparation). The parameters listed in Table 2 indicate that thin-section and MRI data are highly correlated, with a $6 \%$ deviation in total porosity and an average $20 \%$ deviation in the linear dimensions of pores. Consistently, MRI-derived parameters are larger than those derived from the thinsection. This pattern is also evident in the pore-size distribution and in particular the autocorrelation function (acf) shown in Fig. 5. The latter (Fig. 5a) indicates that for lags between 1 and $5 \mathrm{~mm}$, the MRI data are more highly correlated, suggestive of longer, more connected small-scale pores. Similarly, the pore-size distribution exhibits the largest mismatch in the smallest size category (Fig. 5b).

These deviations are in part due to sample artifacts and mismatch in co-location of the two images. However, much more important are the differences in section thickness. The thin-section image can be effectively thought of as two-dimensional (i.e., section thickness $<0.05 \mathrm{~mm}$ ) since the sample surface is recorded in incident light with pores filled with a contrast agent (Eicken et al., in preparation). The MRI data, on the other hand, represent an integral over a volume slice of about $1.3-\mathrm{mm}$ thickness. Hence, pore-size parameters are subject to stereological effects which are likely to result in an overestimate compared to truly two-dimensional data sets (Underwood, 1970; Eicken, 1993). As will be shown below, this problem diminishes with increasing resolution or decreasing slice thickness. The comparative analysis of these two data sets also demonstrated that magnetic field and gradients are homogeneous throughout the sample volume such that image distortions as observed by Edelstein and Schulson (1991) are not affecting data quality.

\subsection{High-resolution imaging of pore microstructure}

\subsubsection{Comparison between thin-section and MRI data}

In columnar, congelation-type sea ice, the bulk of the fluid typically resides within vertical, sub-parallel brine layers few tenths of millimeters wide, oriented perpendicular to the crystals' c-axis (Weeks and
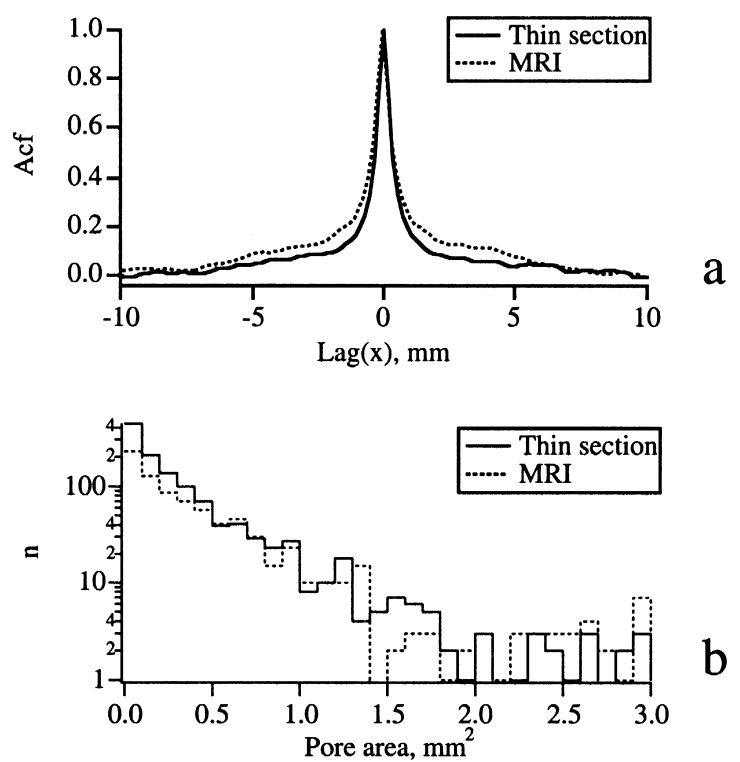

Fig. 5. Comparison of microstructural parameters for thin-section and MRI images shown in Fig. 3 (sample H961219Q, 0.05-m depth): Horizontal cross-section through 2-D acf (Fig. 5a) and pore-size distribution based on cross-sectional pore area (Fig. 5b). 
(a)

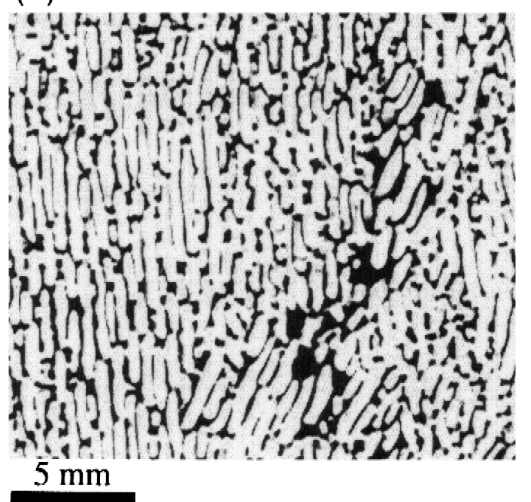

(b)

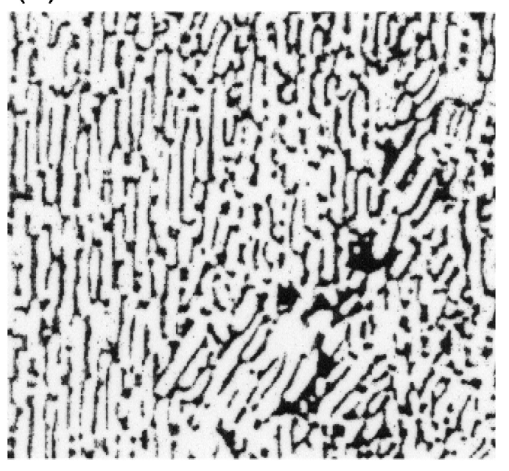

Fig. 6. Comparison of pore microstructure between horizontal thin-section image (a) — averaged over three serial sections spaced 0.2 mm apart - and MRI data recorded with the mini-imaging unit (b) of the same sea-ice sample (H970128Q, 0.15-m depth). Pores appear dark in both images, with system of subparallel brine layers clearly discernible (see Table 3 for further details on image acquisition and pore-size data).

Ackley, 1986). With an FOV extending over close to $100 \mathrm{~mm}$, a pixel resolution of around $0.2 \mathrm{~mm}$ and a slice thickness of $>1 \mathrm{~mm}$ (Table 2), imaging in a field gradient of $50 \mathrm{mT} \mathrm{m}^{-1}$ is ideal for studies of pore macrostructure in a larger sample volume (Figs. 2 and 3), but proved only marginally effective at properly resolving the sub-millimeter pore structure. This is also borne out by the mismatch apparent between thin-section and MRI data at smaller pore sizes in Fig. 5.

A significant increase in resolution and data quality has been achieved, however, by imaging in higher field gradients of up to $200 \mathrm{mT} \mathrm{m}^{-1}$. In combination with the cooling chamber, this system allows for the

Table 3

Comparison between thin-section image and MRI data (sample H970128Q, 0.15-m depth, high-resolution, $200 \mathrm{mT} \mathrm{m}^{-1}$ gradient coils, see Fig. 6)

\begin{tabular}{|c|c|c|}
\hline \multirow[t]{2}{*}{ Parameter } & $\begin{array}{l}\text { Thin section } \\
\text { data }\end{array}$ & MRI data \\
\hline & Mean $\pm \sigma$ & $\operatorname{Mean} \pm \sigma$ \\
\hline Pixel size (horizontal), mm & 0.08 & 0.09 \\
\hline Slice thickness (vertical), mm & $<0.05$ & 0.4 \\
\hline Porosity & 0.175 & 0.181 \\
\hline Pore area, $\mathrm{mm}^{2}$ & $0.32 \pm 1.31$ & $0.28 \pm 0.66$ \\
\hline Pore perimeter, mm & $2.92 \pm 5.80$ & $2.72 \pm 3.93$ \\
\hline Pore major axis, mm & $0.87 \pm 0.90$ & $0.85 \pm 0.82$ \\
\hline Pore minor axis, $\mathrm{mm}$ & $0.30 \pm 0.23$ & $0.29 \pm 0.22$ \\
\hline
\end{tabular}

analysis of samples with approximate dimensions $30 \times 30 \times 60 \mathrm{~mm}$, comparable to high-resolution image analysis of thin sections. While the smaller FOV reduces the SNR, this can be compensated for by longer integration times. Smaller sample sizes also reduce the amount of attenuation of the RF signal
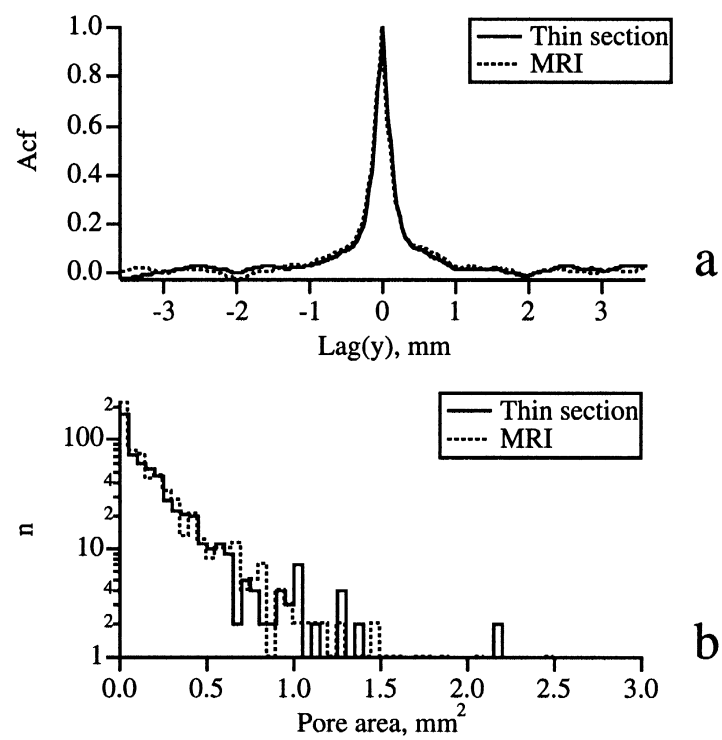

Fig. 7. Comparison of microstructural parameters for thin-section and MRI images shown in Fig. 6 (sample H970128Q, 0.15-m depth). Vertical cross-section through 2-D acf (Fig. 7a) and pore-size distribution based on cross-sectional pore area (Fig. 7b). 

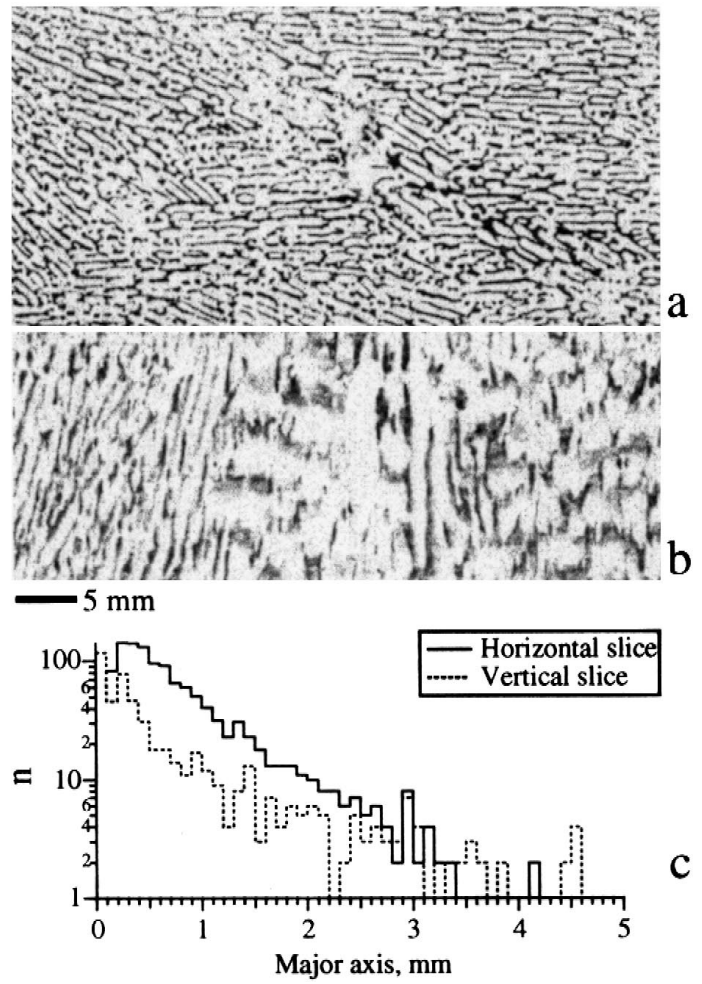

Fig. 8. Horizontal (a) and vertical (b) MRI slices through sample H970128Q, 0.15-m depth grown at zero under-ice current speed (samples centrifuged and saturated with decane at in-situ temperature; pores appear dark). Pore-size distribution for vertical and horizontal slice (major axis of inscribed ellipse) shown in Fig. 8c.

which becomes particularly important in the analysis of sea-ice samples in brine immersion (see Sections 3.1 and 4). Comparison between MRI and thin-section images (Fig. 6) indicates good agreement. This is supported by microstructural data (Table 3), with an average deviation between thin-section and MRIderived linear size parameters of around $4 \%$ and a difference in porosity of $3 \%$. The effects of finite MRI slice thickness as compared to effectively twodimensional section data have been reduced in this case by averaging over a number of thin-section images extending through the full depth of the MRI slice. This favourable comparison is augmented by the close correspondence between acfs derived along the principal (vertical) axis of the predominantly lamellar brine inclusions shown in Fig. 7a. Both the acf as well as the pore-size distribution of the MRI data (Fig. 7b) adhere much closer to the thin-section images than is the case for results obtained with the standard gradient coils (Fig. 5).

\subsubsection{Pore microstructure derived from MRI data obtained at higher gradients (up to $200 \mathrm{mT} \mathrm{m}^{-1}$ )}

The examples of pore microstructure discussed above focussed on coarse-grained columnar sea ice with a well-developed lamellar substructure, grown in the laboratory at zero current speed and near-constant air temperature. In this section, microstructural data of this type of ice will be compared to data from ice grown under contrasting hydrodynamic condi-
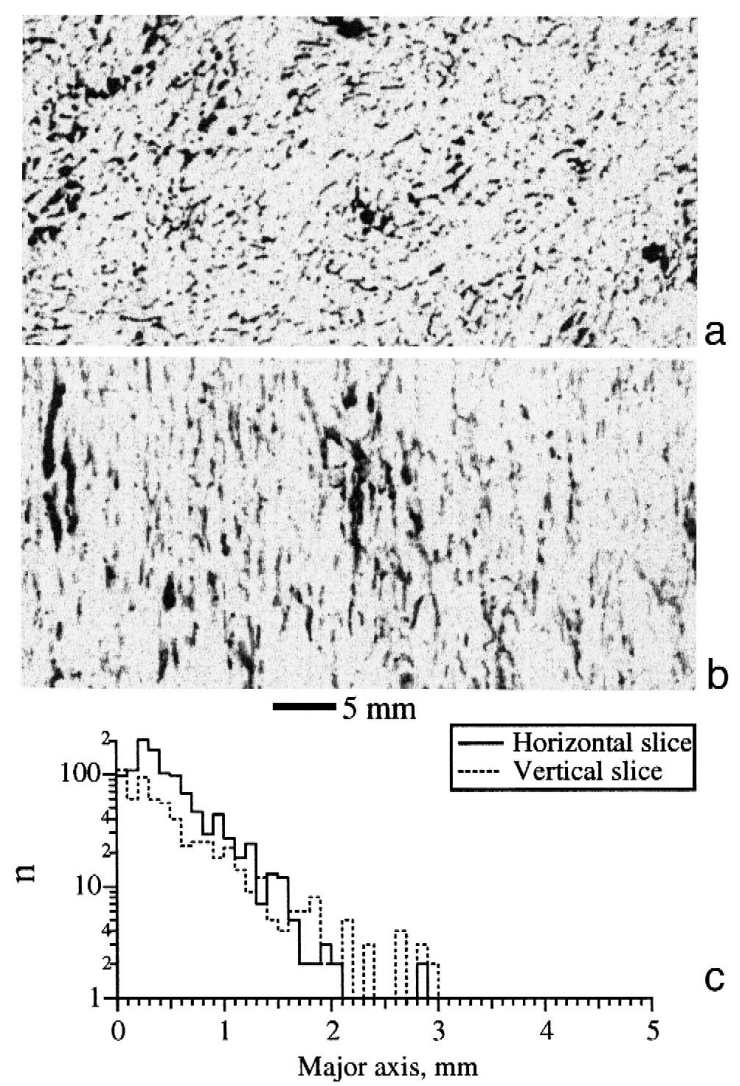

Fig. 9. Horizontal (a) and vertical (b) MRI slices through sample H961219C, 0.08-m depth grown at an under-ice current speed of $0.16 \mathrm{~m} \mathrm{~s}^{-1}$ (current direction from upper left to lower right of sample in horizontal section; samples centrifuged and saturated with decane at in-situ temperature; pores appear dark). Pore-size distribution for vertical and horizontal slice (major axis of inscribed ellipse) shown in Fig. 9c. 
Table 4

Comparison between MRI data (horizontal and vertical sections) for ice grown at different under-ice current speeds (high-resolution imaging at $200 \mathrm{mT} \mathrm{m}^{-1}$, see Figs. 8 and 9; mean and standard deviation for mean values of eight individual sections per sample are shown)

\begin{tabular}{|c|c|c|c|c|}
\hline \multirow[t]{2}{*}{ Parameter } & \multicolumn{2}{|c|}{ H970128Q, $0.15 \mathrm{~m}$} & \multicolumn{2}{|c|}{ H961219C, $0.08 \mathrm{~m}$} \\
\hline & Horizontal & Vertical & Horizontal & Vertical \\
\hline Current velocity, $\mathrm{m} \mathrm{s}^{-1}$ & 0.00 & 0.00 & 0.16 & 0.16 \\
\hline Porosity & $0.21 \pm 0.01$ & $0.20 \pm 0.02$ & $0.11 \pm 0.01$ & $0.11 \pm 0.01$ \\
\hline Pore area, $\mathrm{mm}^{2}$ & $0.27 \pm 0.03$ & $0.43 \pm 0.10$ & $0.14 \pm 0.02$ & $0.25 \pm 0.04$ \\
\hline Pore perimeter, $\mathrm{mm}$ & $2.66 \pm 0.26$ & $2.94 \pm 0.47$ & $1.43 \pm 0.11$ & $1.95 \pm 0.15$ \\
\hline Pore major axis $a_{\text {maj }}, \mathrm{mm}$ & $0.83 \pm 0.06$ & $0.87 \pm 0.13$ & $0.50 \pm 0.03$ & $0.68 \pm 0.03$ \\
\hline Pore minor axis $a_{\min }, \mathrm{mm}$ & $0.30 \pm 0.02$ & $0.30 \pm 0.03$ & $0.24 \pm 0.01$ & $0.25 \pm 0.01$ \\
\hline
\end{tabular}

tions with an under-ice current speed of approximately $0.16 \mathrm{~m} \mathrm{~s}^{-1}$. These measurements are motivated by the lack of data and understanding of the microstructural evolution of sea ice in different hydrodynamic regimes in particular as it relates to pore structure and ice properties. Changes in the microstructural evolution are forced by variability in the thickness and structure of the laminar boundary layer, on the order of a few millimeters thick, ahead of the advancing ice-water interface (Weeks and Ackley, 1986; Wettlaufer, 1998). The MRI data presented here are complementary to a more detailed study of the evolution of pore space as a function of under-ice current speed based mostly on horizontal thin-section data by Eicken et al. (in preparation).
A comparison between the image data shown for an ice cover grown at zero current (Fig. 8) and at an under-ice current speed of approximately $0.16 \mathrm{~m} \mathrm{~s}^{-1}$ (Fig. 9), in the upper range of natural conditions (McPhee, 1990), demonstrates strong contrasts in microstructure. For ice affected by a current of this magnitude, the grain substructure with parallel or sub-parallel brine layers separated by orderly arrays of ice lamellae (Fig. 8a) breaks down into smaller disjoint pores of more isometric cross-section (Fig. 9a). In the vertical plane (Figs. 8b and 9b), this structural change is just as pronounced with the parallel brine layer arrays replaced by shorter (Fig. 9c), less well-ordered brine tubes. Such changes evident in the MRI scenes are reflected in the pore-

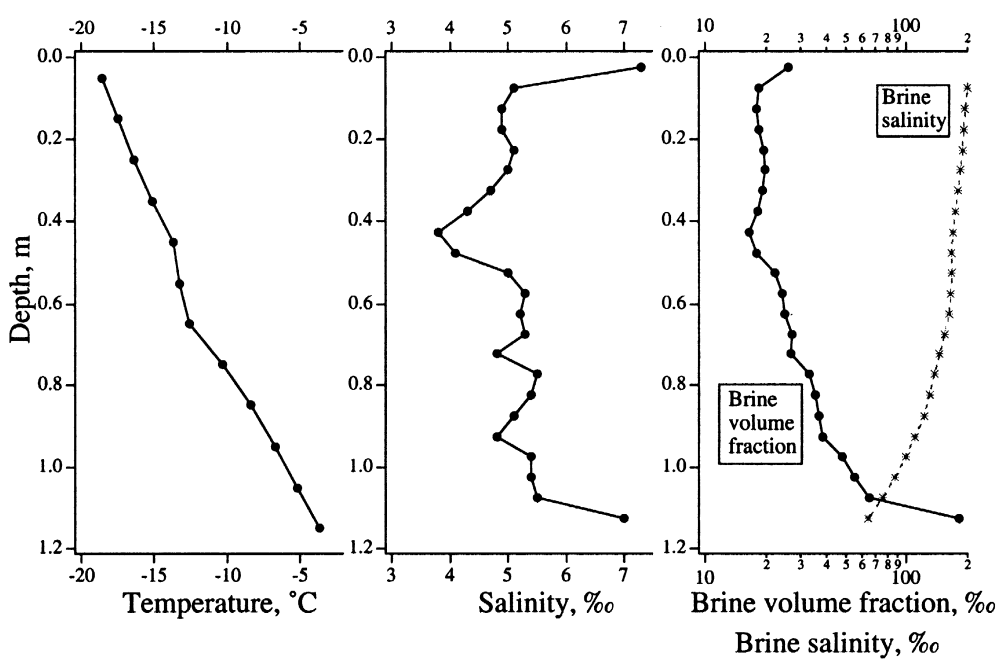

Fig. 10. Profiles of temperature (left), salinity (center) as well as brine volume fraction and brine salinity (right, derived based on phase relations according to Cox and Weeks, 1983) through the fast-ice sheet at the sampling site near Barrow in the Chukchi Sea (March 12, 1999). 

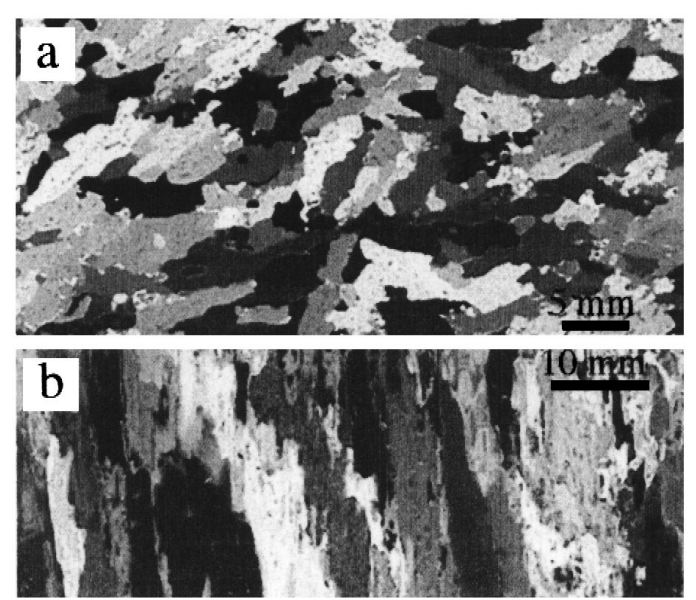

Fig. 11. Horizontal (a) and vertical (b) thin section images through Chukchi Sea ice sample, (March 12, 1999, 0.13- to 0.16-m depth, crossed polarizers).

size distribution (for example in the length of the major axis of an inscribed ellipse shown in Figs. 8c and 9c) derived from these data sets. The ice grown under calm conditions exhibits larger pores (see also Table 4) and a more pronounced anisotropy both within the horizontal plane (not shown) and in com- paring pore maximum dimensions in the horizontal and vertical plane (Table 4). Based on mostly qualitative information published on the effects of hydrodynamics on grain and pore sizes, it appears as if the coarsening and homogenization observed at moderate current speeds (Weeks and Gow, 1978; Weeks, 1998) break down at higher flow velocities.

\section{Results: thermal evolution of pore microstruc- ture in natural sea ice}

The fact that non-destructive tomographic techniques such as MRI allow for direct studies of pore-space evolution as a function of thermal forcing is a particular strength of the method. The overall increase in fractional brine volume with temperature is dictated by the thermodynamic phase relations and, given the salinity and temperature of an ice volume and assuming thermodynamic equilibrium, can be derived from semi-empirical relationships (Assur, 1960; Cox and Weeks, 1983) or from geochemical models (Spencer et al., 1990). The corresponding changes in the size and morphology of the
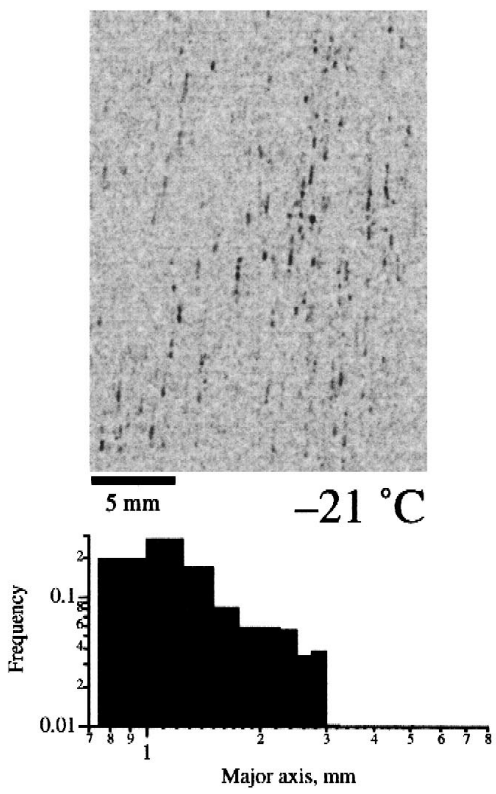
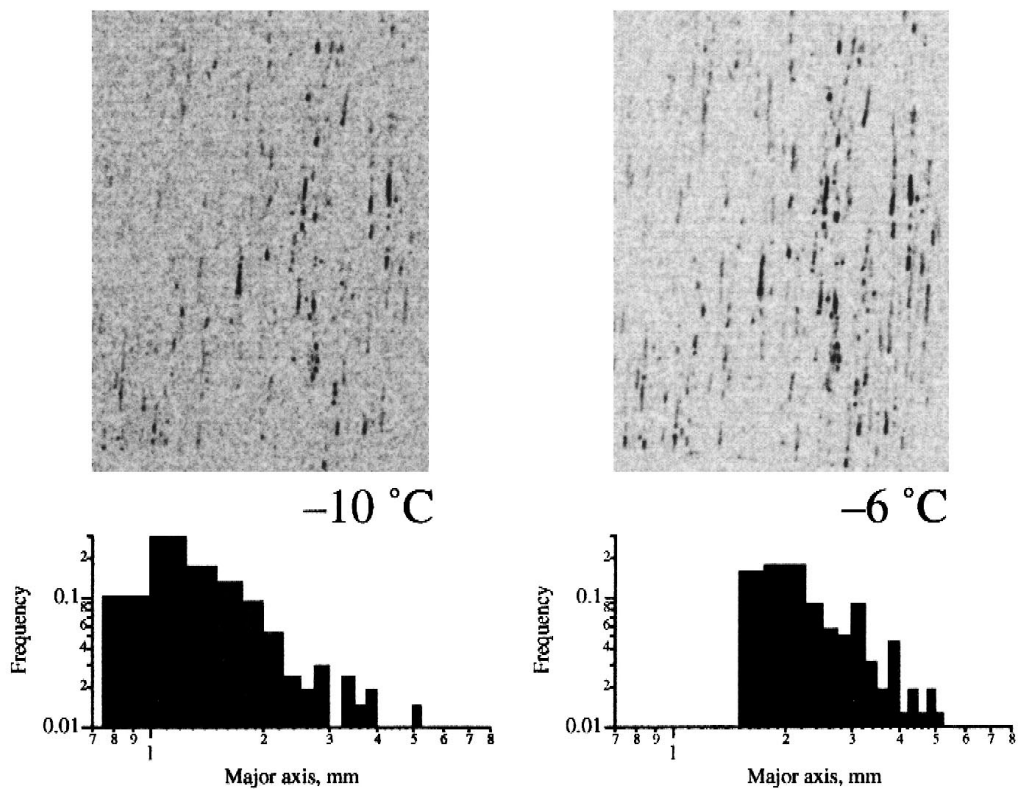

Fig. 12. Thermal evolution of pores in Chukchi Sea ice sample (CS980312, 0.13- to 0.16-m depth, see Fig. 10 for profile properties and Fig. 11 for thin-section images). Shown at the top are the MRI data (vertical slice) at the temperatures indicated above. Below, the size distribution of the upper 10-percentile of the major axes of pores $\left(a_{\mathrm{maj}}\right)$ in all the vertical slices is shown. 
fluid inclusions cannot be arrived at in such a fashion, however. The few observations of changes in pore structure are either based on semi-quantitative microscopy (Assur, 1960) or measurements carried out on larger populations of samples (Perovich and Gow, 1996).

Here, we have studied changes in pore microstructure associated with a warming from below $-21^{\circ} \mathrm{C}$ to $-6^{\circ} \mathrm{C}$ throughout the same, undisturbed ice sample volume. Samples had been obtained from the coastal fast-ice cover in the eastern Chukchi Sea near Barrow during the coldest weeks of the year (Section 2.2). Temperatures were maintained at or close to the in-situ temperature which approached $-20^{\circ} \mathrm{C}$ in the upper ice layers (Fig. 10) up to the start of the MRI analysis. Salinity measurements and microstructural studies performed on additional ice cores obtained at the study site indicate the ice cover to consist of prototypical first-year columnar, congelation-type sea ice with a $\mathrm{C}$-shaped salinity profile
(Figs. 10 and 11), which is in agreement with earlier studies carried out at this location (Weeks and Gow, 1978; Cole and Shapiro, 1998). The MRI sample was obtained from the cold, uppermost layers of columnar ice, composed of smaller crystals and lacking the preferred azimuthal alignment of crystallographic c-axes displayed at greater depths (Fig. 11). Based on the salinity and temperature measurements, the brine volume fraction in the upper layers of columnar sea ice is approximately 20\%o (Fig. 10, derived according to Cox and Weeks, 1983). The corresponding MRI data shown in Fig. 12 and Table 5 are in fair agreement with this bulk brine porosity.

The most interesting aspects of Fig. 12, however, are the changes in pore microstructure associated with a warming of the sample from $-21^{\circ} \mathrm{C}$ to $-6^{\circ} \mathrm{C}$. As is clearly evident, pores present at low temperatures enlarge and join, while at the same time new populations of pores appear in the image data (see Table 5, pore number densities). The latter are

Table 5

Thermal evolution of pore microstructural parameters as derived from MRI data for sample CS990312, 0.10- to 0.13-m depth (bold numbers in upper rows are mean values, standard deviation indicated in lower rows)

\begin{tabular}{|c|c|c|c|c|c|c|c|c|c|}
\hline \multirow[t]{2}{*}{$T\left({ }^{\circ} \mathrm{C}\right)$} & \multicolumn{3}{|c|}{ Vertical sections } & \multicolumn{3}{|c|}{ Horizontal sections } & \multirow[t]{2}{*}{$P$} & \multirow[t]{2}{*}{$N_{\mathrm{P}, \text { ver }}\left(\mathrm{mm}^{-3}\right)$} & \multirow[t]{2}{*}{$N_{\mathrm{P}, \text { hor }}\left(\mathrm{mm}^{-3}\right)$} \\
\hline & $a_{\mathrm{maj}}(\mathrm{mm})$ & $a_{\min }(\mathrm{mm})$ & $r_{\text {maj } / \min }$ & $a_{\mathrm{maj}}(\mathrm{mm})$ & $a_{\min }(\mathrm{mm})$ & $r_{\text {maj } / \min }$ & & & \\
\hline \multicolumn{10}{|c|}{ Entire data set } \\
\hline-21 & 0.47 & 0.10 & 1.10 & 0.19 & 0.09 & 0.56 & & & \\
\hline \multicolumn{10}{|c|}{ Top 10 percentile } \\
\hline \multicolumn{10}{|c|}{ Entire data set } \\
\hline \multirow{2}{*}{-10} & 0.43 & 0.22 & 1.73 & 0.34 & 0.23 & 1.46 & 0.027 & 0.62 & 1.05 \\
\hline & 0.52 & 0.10 & 1.18 & 0.22 & 0.10 & 0.57 & & & \\
\hline \multicolumn{10}{|c|}{ Top 10 percentile } \\
\hline \multirow[t]{2}{*}{-6} & 0.70 & 0.26 & 2.32 & 0.38 & 0.25 & 1.53 & 0.038 & 0.58 & 1.08 \\
\hline & 0.79 & 0.13 & 1.74 & 0.24 & 0.11 & 0.59 & & & \\
\hline \multicolumn{10}{|c|}{ Top 10 percentile } \\
\hline \multirow[t]{2}{*}{-6} & 2.62 & 0.45 & 6.16 & 0.92 & 0.42 & 2.32 & & & \\
\hline & 1.06 & 0.15 & 2.37 & 0.34 & 0.12 & 0.84 & & & \\
\hline
\end{tabular}

$a_{\text {maj }}, a_{\text {min }}:$ major and minor axis of inscribed ellipse, $r_{\text {maj/min }}:$ ratio between major and minor axis, $P:$ porosity, $N_{\mathrm{P}, \mathrm{ver}}, N_{\mathrm{P}, \mathrm{hor}}:$ number density of pores. 
assumed to represent pores that were not detectable due to their small size in the data obtained at lower temperatures. The pore-size distributions derived from segmented MRI data support this conclusion and allow for further scrutiny of these changes. Thus, despite the appearance of new pore populations, the number density (or total number of pores in the sample) actually decreases by approximately one quarter in the vertical slices over the entire temperature interval. This is a result of the joining of disjunct pores during warming and is reflected in particular by the overall increase in the size of the largest 10-percentile of the pore population (Fig. 12, bottom). A comparison between the size of the vertical axis of pores and the two major and minor horizontal axes for different temperatures furthermore demonstrates that the increase in pore size occurs mostly in the vertical direction, with the vertical major axis dimension increasing by a factor of more than 1.5 between $-10^{\circ} \mathrm{C}$ and $-6^{\circ} \mathrm{C}$ and the horizontal axes increasing by less than 1.15 over the same temperature interval. Corresponding patterns are observed in the pore size distributions (Table 5). These increases in pore size are accompanied by changes in pore shape, with increasing elongation in the vertical as compared to the horizontal direction (Table 5).

\section{Discussion}

\subsection{An assessment of strengths and potential sources of error for MRI sea-ice data sets}

As demonstrated by the close correspondence between the thin-section images and the corresponding MRI data shown in Figs. 3 and 7, the system employed in this study is capable of rendering the pore space geometry of sea ice to a degree of accuracy and resolution that is comparable to high-quality thin-section imaging. In particular at higher gradients of $200 \mathrm{mT} \mathrm{m}^{-1}$, the data are not compromised by resolution and distortion problems reported in a previous MRI study of sea ice (Edelstein and Schulson, 1991). All of these measurements can be carried out in a non-destructive fashion within the entire range of natural sea-ice temperatures between $0^{\circ} \mathrm{C}$ and $-25^{\circ} \mathrm{C}$, hence, allowing for studies of the thermal evolution of sea-ice brine inclusions that are not hampered by some of the problems of conventional thin-section analysis.

A significant limitation in the application of MRI to sea-ice problems is posed by the strong absorption of RF signals in the lossy brine. As a result, both the SNR and the resolution are significantly repressed in comparison with inclusions of other, non-polar fluids devoid of ionic impurities. This is less of a problem at lower temperatures or lower salinities, where the fractional pore volume is below $50 \%$ o to $100 \%$, but can become increasingly problematic for highly porous samples at warmer temperatures, such as the example shown in Fig. 2. In such cases, the SNR decreases and warming of the sample cannot be avoided over longer measurement intervals. This results in an effective degrading of the resolution, which foils most studies of the geometry of the primary, small-scale pore space. Apart from increasing the field gradients, a promising strategy for imaging of pores in the high-porosity, high-permeability regime is to replace the brine with a contrast agent such as decane. Employing both these methods, the differences between thin-section and MRIderived average linear pore dimensions is reduced to less than 3\%. Part of these differences are attributed to problems in saturating the sample with the contrast agent (see, e.g., the apparent reduction in the size of a larger pore conspicuous in the lower right of the sample shown in Fig. 6 due to incomplete filling of pore with decane) or lining up corresponding thin-section and MRI scenes, whose linear image dimensions may differ by a few percent as a result of a residual mismatch after applications of affine image transforms.

The errors associated with the segmentation process that separates the fluid inclusions from the solid ice matrix are considerably smaller than in comparable thin-section studies because of the sensitivity of the NMR signal to the fractional brine volume. Thus, the maximum-likelihood classification error amounts to less than $3 \%$ (based on fractional volume of pores, for average linear pore dimensions it is below 1\%) for an assumed maximum error in the derivation of the segmentation threshold by $10 \%$.

The factor of largest significance for the interpretation of MRI data, in particular in comparison with thin-section images of the sample surface taken in 
incident light, is the slice thickness, which may be larger than the characteristic pore dimension. Slice thicknesses for MRI data measured with the standard gradient coils are generally larger than $1 \mathrm{~mm}$ (depending on matrix size, FOV and available RF power) and even a data set optimized for resolution is likely to have slice thicknesses larger than $0.3 \mathrm{~mm}$. This introduces stereological effects (Underwood, 1970; Eicken, 1993) that affect the apparent pore size and to a lesser extent, for columnar sea ice, the apparent shape of the inclusions.

The close spacing between individual slices, such as a set of eight separated by approximately $4 \mathrm{~mm}$ each for the samples shown in Figs. 8 and 9, allows an assessment of the spatial variability of pore volume and microstructure at a very small scale. The standard deviation of the slice porosities varies between $7 \%$ and $12 \%$ for these two different samples (Table 4), with maximum deviations of $20 \%$, corresponding to maximum bulk salinity differences of approximately $1.4 \%$ o salinity. Microstructural size parameters also exhibit standard deviations of 5-10\% between sets of horizontal or vertical slices. These fluctuations are most likely associated with grainboundaries and other microstructural discontinuities in the sample.

\subsection{The thermal evolution of sea-ice pore space}

In contrast with destructive imaging techniques such as standard thin-section processing, MRI can provide imagery of a larger sea-ice sample as it undergoes temperature changes. Here, these changes have been documented for a sample of columnar ice maintained close to in-situ temperatures after coring and then warmed from $-21^{\circ} \mathrm{C}$ to $-6^{\circ} \mathrm{C}$. As shown in Fig. 12 and Table 5 and summarized in Fig. 13, warming was accompanied by significant microstructural changes, in particular between $-10^{\circ} \mathrm{C}$ and $-6^{\circ} \mathrm{C}$. Concurrently, pore volumes increased by approximately $50 \%$, which is in good agreement with theoretical estimates based on the ice salinity and thermodynamic phase relations (Fig. 13). As expected, based on earlier semi-quantitative observations on thin sections (Assur, 1960, Weeks and Ackley, 1986), the vertical elongation of pores increases as a result of warming, with the ratio between major and minor axis (inscribed ellipse)

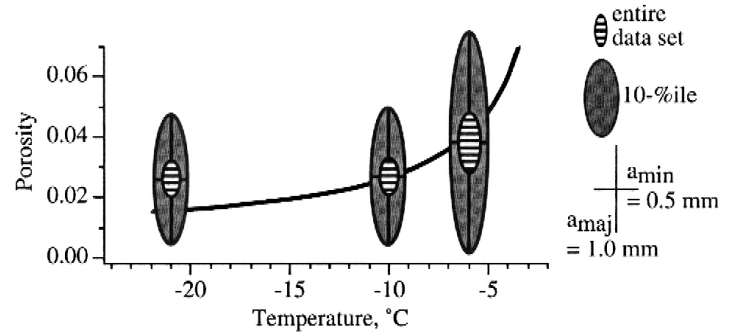

Fig. 13. Thermal evolution of fluid inclusions for Chukchi Sea ice sample (CS990312, 0.13- to 0.16-m depth, see Fig. 10 for profile properties and Fig. 11 for thin-section images) based on MRI data. The relative proportions of the major and minor pore axis dimensions are shown for the entire data set and the upper 10-percentile (scale shown at right). The solid line denotes the increase in brine porosity based on sample bulk salinity and phase relations.

$r_{\text {maj/min }}$ increasing from 4 to more than 6 for the upper 10-percentile of pores in a sample. During the same temperature interval, $r_{\text {maj/min }}$ increased by less than $10 \%$ to $2.3 \%$ for the upper 10-percentile in horizontal MRI slices. Based on the mean parameters, the aspect ratios of brine inclusions in three dimensions (vertical, horizontal parallel to crystal basal layer, horizontal perpendicular to basal layer) have thus been observed to increase from 1.9:1.5:1 to $2.8: 1.5: 1$ for the entire pore population and from 4.1:2.0:1 to 6.2:2.2:1 for the upper 10-percentile.

With increasing size, pores have been observed to merge and coalesce (Fig. 14). While this circumstance is also reflected in a decrease in the number density of pores $N_{\mathrm{P}}$ in vertical MRI slices, the appearance of new populations of small pores in the image data has diminished the overall magnitude of this effect. Fig. 14 illustrates the problem and also shows that the assessment of pore morphology (e.g., $\left.r_{\text {maj/min }}\right)$ is to some extent compromised as well. Thus, the merging of four apparently isolated pores into a large, tubular inclusion in the center is accompanied by the "appearance" of new pores to the right and above this major feature. The latter are responsible for an increase in $N_{\mathrm{P}}$ for this small sub-scene. In the horizontal MRI slices, this process is believed to be responsible for the slight increase observed for $N_{\text {P,hor }}$ with increasing temperature (Table 5). A similar, and upon first reflection counter-intuitive trend was reported by Perovich and Gow (1996) in their analysis of thin-section images of warming ice sam- 


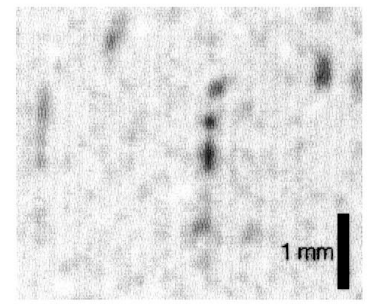

$-21^{\circ} \mathrm{C}$

$\mathrm{N}_{\mathrm{p}}: 0.36 \mathrm{~mm}^{-3}$

$\mathrm{a}_{\text {maj: }}: 0.45 \mathrm{~mm}$

$\max \left(\mathrm{a}_{\mathrm{maj}} / \mathrm{a}_{\min }\right): 3.3$

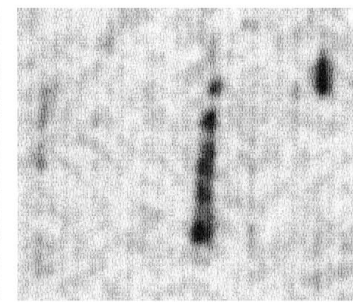

$-10{ }^{\circ} \mathrm{C}$

$\mathrm{N}_{\mathrm{p}}: 0.36 \mathrm{~mm}^{-3}$

$\mathrm{a}_{\text {maj: }}: 0.78 \mathrm{~mm}$

$\max \left(a_{\operatorname{maj}} / a_{\min }\right): 7.5$

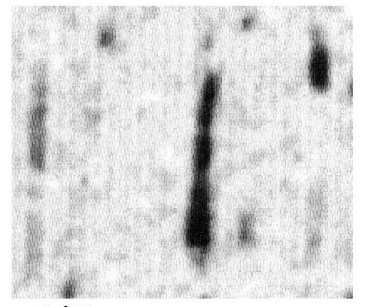

$-6^{\circ} \mathrm{C}$

$\mathrm{N}_{\mathrm{p}}: 0.71 \mathrm{~mm}^{-3}$

$\mathrm{a}_{\text {maj: }}: 0.78 \mathrm{~mm}$

$\max \left(\mathrm{a}_{\operatorname{maj}} / \mathrm{a}_{\min }\right): 8.3$

Fig. 14. Thermal evolution of individual brine inclusions in vertical MRI slice (sample CS990312, approximately 0.15-m depth; detail from images shown in Fig. 12, center; original pixel data interpolated onto smaller grid). Number density of pores $N_{\mathrm{P}}$, mean length of major pore axis $a_{\text {maj }}$ and maximum ratio between major and minor pore axis length max $\left(a_{\mathrm{maj}} / a_{\mathrm{min}}\right)$ also indicated.

ples (horizontal sections only). Analogous to the Perovich and Gow study, this phenomenon is to a large degree a result of the limitations in image resolution in the case of MRI data sets rather than the manifestation of newly nucleated brine inclusions. The pores appearing in the $-6^{\circ} \mathrm{C}$ image in Fig. 14 are actually on the order of one pixel in size (note that the image has been enhanced through interpolation) and have hence not been detected as separate entities at lower temperatures and correspondingly smaller volume fractions. The introduction of small pores into higher-temperature images furthermore introduces a significant bias towards isometric, non-elongated pores which explains the differences in pore elongation between the entire data set and the upper 10-percentile (Table 5). In the example shown in Fig. 14, mean pore elongation increases by 1.7 during warming, whereas the increase for the largest pore amounts to 2.5.

A detailed assessment of the effects of resolution on microstructural parameters is beyond the scope of this contribution, but we would like to point out three important ramifications. First, as is well-established in the context of the fractal geometry of microstructure (Dearnley, 1985; Eicken, 1991), the resolution-dependence of some geometric descriptors can significantly compromise comparisons between data sets collected at different resolution or employing different methods. For instance, Eicken (1991) has described 1.5- to 2-fold increases in the derived grain-boundary density for an increase in image resolution by a factor of 3 . Taking these disparaties into account, the present MRI pore data set is quite comparable to the pore size and density data collected by Perovich and Gow (1996). At a pixel resolution of approximately $0.03 \mathrm{~mm}$, they measured pore number densities $N_{\mathrm{P} \text {,hor }}$ between 0.2 and 2.2 $\mathrm{mm}^{-3}$ and major axis lengths between 0.24 and 0.63 $\mathrm{mm}$ at comparable temperatures on horizontal sections of first-year ice. As high-resolution microscopic studies indicate (Light and Maykut, 1999; Eicken et al., 1999), $N_{\mathrm{P}}$ can increase to values well above 100 with corresponding decreases in pore-size parameters.

A second aspect of the problem relates to the fact that pixel dimensions are typically smaller than slice thicknesses in MRI data sets which may in large part explain the contrasts in the thermal evolution of $N_{\mathrm{P}}$ with increasing temperature for horizontal and vertical slices. The preferred vertical alignment of pores renders them more easily detectable in horizontal slices, where vertical pore dimensions exceed the slice thickness of approximately $0.4 \mathrm{~mm}$ at smaller fractional brine volumes than the horizontal pore dimensions do in vertical slices. The slice/pixel geometry may also affect the interpretation of highporosity samples, such as those shown in Figs. 8 and 9. The sub-parallel brine layers in Fig. $8 \mathrm{~b}$ are rendered as distinct horizontal bands in the right half of the image because the finite slice thickness intersects the slightly tilted brine layer over a considerable vertical extent. Here, only truly three-dimensional data or variational slice thicknesses can provide more detailed insight into actual pore geometries. 
Finally, the issue of resolution also affects interpretation of MRI data in the context of pore connectivity and fluid transport. Thus, the interpretation of the merging of individual pores discussed above and shown in Fig. 14 only applies to pore connections visible at the smallest scale resolved in the data set. At sub-pixel scales, the set of pores barely discernible at $-21^{\circ} \mathrm{C}$ in Fig. 14 may already have been linked through smaller-scale vein networks. Given some of the limitations of both MRI and ordinary thin-section analysis, we suspect that only true microscopic analysis may provide deeper insight into this problem. The relevance of this question far exceeds the merely technical aspects of microstructural analysis, however. While the finding that pores are preferentially elongating and in particular merging in the vertical direction is compatible with classical sea-ice pore microstructural models, all of which are essentially variants of the one described by Assur (1960) and Weeks and Ackley (1986), the physical mechanism for the preferential elongation is not completely resolved. Under natural conditions and for larger pores, convective processes are likely to enhance enlargement of pores in the vertical direction (Niedrauer and Martin, 1979; Wettlaufer, 1998). To a lesser extent, migration of inclusions in a temperature gradient may also favour elongation of pores. The warming experiment in this study was conducted in the absence of macroscopic temperature gradients, however, with both the top and the bottom of the sample maintained at the same temperature (Fig. 1). The anisotropy in the ice lattice structure and the corresponding differences in free surface energy parallel and normal to the crystal c-axis can only explain significant shape anisotropy in the kinetically controlled regime of phase change, such as during the growth phase of sea ice (Weeks and Ackley, 1986; Wettlaufer, 1998). The equilibrium shape of brine inclusions, on the other hand, is dominated by the temperature-entropy term in the equation of state and hence, less susceptible to crystallographic lattice anisotropy.

This leaves another explanation that centers on the possibility that the presence of impurities in liquid or quasi-liquid form, in particular along grain and sub-grain boundaries, may be sufficient to foster invasion of these impurity-rich zones by brine during warming of the sample. If such proto-inclusions were to persist at lower temperatures they would provide pathways (possibly on a sub-pixel scale in the context of this MRI study) for an influx of brine upon warming to higher temperatures. In this context, the present work can only be the first step in elucidating the linkages between impurity content, microstructure and fluid flow in sea ice. MRI methods, in particular in conjunction with optical microscopy, appear to hold great promise in providing deeper insight into the thermal evolution of sea-ice fluid inclusions.

\section{Summary and conclusions}

MR imaging has been successfully applied to the high-resolution study of brine inclusions in sea ice. Samples were maintained at temperatures between $-2^{\circ} \mathrm{C}$ and $-25^{\circ} \mathrm{C}$ with the help of a flow-through cooling unit. At field gradients of $50 \mathrm{mT} \mathrm{m}^{-1}$, the dielectric properties of brine with high loss factors at frequencies of few hundred $\mathrm{MHz}$ resulted in a significant degradation of the signal, heating of the sample due to high RF power and reduced image resolution (pixel sizes $>0.2 \mathrm{~mm}$, slice thicknesses $>1 \mathrm{~mm}$ ). These resolution problems, which had been encountered in earlier work, were overcome through the use of decane as a contrast agent and operation at higher gradients.

Analysis of MRI data of different types of artificial sea ice grown under controlled conditions in an environmental test basin and comparison with conventional thin-section image analysis indicates good to very good agreement between the two methods. Under optimal conditions, MRI data can be considered equivalent or superior to thin-section data at a comparable resolution $(<0.1-\mathrm{mm}$ pixel size, slice thickness $<0.4 \mathrm{~mm})$. Moreover, development of a segmentation technique based on the liquid fraction within each volume element allows for physically based, reliable discrimination between pores and ice matrix in the data sets.

Analysis of ice samples grown at zero and high under-ice current speeds reveal distinct differences in pore microstructure as a function of current speed. The lamellar microstructure of brine inclusions evident at zero current speeds is much less pronounced at current speeds of $0.16 \mathrm{~m} \mathrm{~s}^{-1}$, with a correspond- 
ing decrease in pore size by more than one third. While it is well-established that crystal alignment increases with moderate current speeds (Weeks and Gow, 1978; Weeks, 1998), the present results indicate that the concurrent homogenization of grain and pore structure breaks down at higher current speeds. Work to elucidate linkages between pore and grain microstructure and a more detailed discrimination of the dependence of microstructure on the under-ice hydrodynamic regime is currently underway (Eicken et al., in preparation).

The thermal evolution of brine inclusions in natural sea ice, obtained at low ambient temperatures and maintained at close to in-situ temperatures after sampling, was studied between $-21^{\circ} \mathrm{C}$ and $-6^{\circ} \mathrm{C}$. Aided by the non-destructive nature of MRI and equable environmental conditions during processing and storage of ice samples, we have obtained a data set manifesting the significant changes in pore size, shape and connectivity during ice warming, in particular between $-10^{\circ} \mathrm{C}$ and $-6^{\circ} \mathrm{C}$. Here, mean pore size as well as elongation increase while pore number densities decrease as a result of merging and coalescence of pores in the vertical direction. At the same time, these changes are to some extent compensated by the appearance of new populations of smaller pores that increase in size to above the resolution limit. The present experiments allow first conclusions about the role of impurities along (sub)grain boundaries in promoting the pronounced vertical elongation of pores during warming. Future work, most likely through a combination of MRI and optical microscopy will have to elucidate the details of these processes and help further our understanding of the critical transition between the low- and highpermeability regimes of sea ice as a porous medium.

\section{Acknowledgements}

Financial support by the National Science Foundation (Grants OPP-9817738 and OPP-9813221) is gratefully acknowledged. The MRI measurements at the Alfred Wegener Institute were supported by a grant from the German Ministry of Research (BEO/BMBF, Project 03PL02A). INTERICE experiments were funded by the European Union's Large Scale Facility Program and benefitted substantially from the support of the director and staff of the Ice Tank Facilities at the Hamburgische SchiffbauVersuchsanstalt. Thanks to the support by the Barrow Arctic Science Consortium, and in particular Dave Ramey, the field work was accomplished smoothly and effectively at Barrow. Further help from Christopher Krembs, Karen Junge, Lew Shapiro (who along with an anonymous reviewer also provided helpful comments on the manuscript), Dave Cole and Fernando Valero Delgado is gratefully acknowledged. H.E. benefitted from collaboration and discussions with Jody Deming, Lew Shapiro and Dave Cole.

\section{References}

Callaghan, P.T., 1991. Principles of Nuclear Magnetic Resonance Microscopy. Clarendon Press, Oxford.

Callaghan, P.T., Dykstra, R., Eccles, C.D., Haskell, T.G., Seymour, J.D., 1999. A nuclear magnetic resonance study of Antarctic sea ice brine diffusivity. Cold Reg. Sci. Technol. 29, 153-171.

Cole, D.M., Shapiro, L.H., 1998. Observations of brine drainage networks and microstructure of first-year sea ice. J. Geophys. Res. 103, 21739-21750.

Cottier, F., Eicken, H., Wadhams, P., 1999. Linkages between salinity and brine channel distribution in young sea ice. J. Geophys. Res. 104, 15859-15871.

Cox, G.F.N., Weeks, W.F., 1983. Equations for determining the gas and brine volumes in sea-ice samples. J. Glaciol. 29, 306-316.

Cox, G.F.N., Weeks, W.F., 1986. Changes in the salinity and porosity of sea-ice samples during shipping and storage. J. Glaciol. 32, 371-375.

Dearnley, R., 1985. Effects of resolution on the measurement of grain 'size'. Mineral. Mag. 49, 539-546.

Deming, J.W., Huston, A.L. An oceanographic perspective on microbial life at low temperatures with implications for polar ecology, biotechnology and astrobiology. In: Seckbach, J. (Ed.), Cellular Origins and Life in Extreme Habitats. Kluwer Publishers, Dordrecht, in press.

Edelstein, W.A., Schulson, E.M., 1991. NMR imaging of saltwater ice. J. Glaciol. 37, 177-180.

Eicken, H., 1991. Quantification of sea-ice properties: automated image analysis of thin sections and parameterization of chlorophyll and salinity distributions (in German). Ber. Polarforsch. $82,1-105$.

Eicken, H., 1993. Automated image analysis of ice thin sections - instrumentation, methods and extraction of stereological and textural parameters. J. Glaciol. 39, 341-352.

Eicken, H. et al., 1998. Ice tank studies of physical and biological sea-ice processes. In: Shen, H.T. (Ed.), Ice in Surface Waters. 
Proceedings of the 14th International Symposium on Ice, Potsdam, New York, USA, 27-31 July. A.A. Balkema, Rotterdam, pp. 363-370.

Eicken, H., Haas, C., Valero Delgado, F. Laboratory experiments on the dependence of sea-ice microstructure on the under-ice current speed, in preparation.

Eicken, H., Stierle, A., Bock, C., Miller, H., Junge, K., Krembs, C., Deming, J., 1999. Morphology and microphysics of sea-ice brine inclusions and their importance for fluid transport and microbial activity. Eos, Trans. Am. Geophys. Union 80, F36.

Freitag, J., 1999. The hydraulic properties of Arctic sea ice implications for the small-scale particle transport (in German). Ber. Polarforsch. 325, 1-150.

Golden, K.M., Ackley, S.F., Lytle, V.I., 1998a. The percolation phase transition in sea ice. Science 282, 2238-2241.

Golden, K.M. et al., 1998b. Forward electromagnetic scattering models for sea ice. IEEE Trans. Geosci. Remote Sens. 36, 1655-1674.

Hallikainen, M., Winebrenner, D.P., 1992. The physical basis for sea ice remote sensing. In: Carsey, F.D. (Ed.), Microwave Remote Sensing of Sea Ice. Geophysical Monograph, vol. 68, American Geophysical Union, Washington, pp. 29-46.

Hannke, S., 1994. High-resolution density measurements on Arctic sea-ice cores with X-rays techniques (in German). Unpublished Undergraduate Thesis, University of Munich and Alfred Wegener Institute, Bremerhaven, Bremerhaven.

Junge, K., Krembs, C., Deming, J., Stierle, A., Eicken, H. A microscopic approach to investigate bacteria under in-situ conditions in sea-ice samples. Ann. Glaciol., 33, in press.

Kawamura, T., 1990. Nondestructive, three-dimensional density measurements of ice core samples by X-ray computed tomography. J. Geophys. Res. 95, 12407-12412.

Kawamura, T., 1988. Observations of the internal structure of sea ice by X-ray computed tomography. J. Geophys. Res. 93, 2343-2350.

Kovacs, A., Morey, R.M., Cox, G.F.N., Valleau, N.C., 1987. Electromagnetic property trends in sea ice: Part I. U.S.A. Cold Reg. Res. Eng. Lab., Hanover, NH CRREL Rep. 87-6:1-45.

Lange, M.A., 1988. Basic properties of Antarctic sea ice as revealed by textural analysis of ice cores. Ann. Glaciol. 10, 95-101.

Light, B., Maykut, G.A., 1999. Observations of sea-ice microstructure. Eos, Transact. Am. Geophys. Union 80, F222.

Lindquist, W.B., Lee, S.-M., Coker, D.A., Jones, K.W., Spanne, P., 1996. Medial axis analysis of void structure in three-dimensional tomographic images of porous media. J. Geophys. Res. 101, 8297-8310.

Melnichenko, N.A., Mikhaylov, V.I., Chizhik, V.I., 1979. Study of the temperature-dependence of the brine content in sea ice by the pulsed NMR method. Oceanology 19, 535-537.

Niedrauer, T.M., Martin, S., 1979. An experimental study of brine drainage and convection in young sea ice. J. Geophys. Res. 84, 1176-1186.

Perovich, D.K., 1998. Optical properties of sea ice. In: Leppäranta, M. (Ed.), Physics of Ice-Covered Seas, vol. 1, Univ. of Helsinki, Helsinki, pp. 195-230.

Perovich, D.K., Gow, A.J., 1996. Quantitative description of sea ice inclusions. J. Geophys. Res. 101, 18327-18343.

Rasband, W.S., Bright, D.S., 1995. NIH Image: a public domain image processing program for the Macintosh. J. Microbeam Analysis 4, 137-149.

Richardson, C., 1976. Phase relationships in sea ice as a function of temperature. J. Glaciol. 17, 507-519.

Richardson, C., Keller, E.E., 1966. The brine content of sea ice measured with a nuclear magnetic resonance spectrometer. J. Glaciol. 6, 89-100.

Schwarz, J., Frederking, R.M.W., Gavrilo, V.P., Petrov, I.G., Hirayama, K.-I., Mellor, M., Tryde, P., Vaudrey, K.D., 1981. Standardized testing methods for measuring mechanical properties of sea ice. Cold Reg. Sci. Technol. 4, 245-253.

Takashima, H., Yamakoshi, H., Maeda, T., Sakurai, A., 1992. Measurement of dielectric constant of model ice (Abstract). Proc. NIPR Symp. Polar Meteorol. Glaciol. 6, 160.

Underwood, E.E., 1970. Quantitative Stereology. Addison-Wesley, Reading, MA.

Weeks, W.F., 1998. Growth conditions and the structure and properties of sea ice. In: Leppäranta, M. (Ed.), Physics of Ice-Covered Seas, vol. 1, Univ. of Helsinki, Helsinki, pp. 25-104.

Weeks, W.F., Ackley, S.F., 1986. The growth, structure and properties of sea ice. In: Untersteiner, N. (Ed.), The Geophysics of Sea Ice. NATO ASI Ser., Ser B 146. Martinus Nijhoff Publ., Dordrecht, pp. 9-164.

Weeks, W.F., Gow, A.J., 1978. Preferred crystal orientations along the margins of the Arctic Ocean. J. Geophys. Res. 84, 5105-5121.

Wehrli, F.W., 1988. Principles of magnetic resonance. In: Stark, D.D., Bradley, W.G. (Eds.), Magnetic Resonance Imaging. Mosby Year Book, St. Louis, pp. 3-20.

Weissenberger, J., Dieckmann, G., Gradinger, R., Spindler, M., 1992. Sea ice: a cast technique to examine and analyze brine pockets and channel structure. Limnol. Oceanogr. 37, 179-183.

Wettlaufer, J., 1998. Introduction to crystallization phenomena in natural and artificial sea ice. In: Leppäranta, M. (Ed.), Physics of Ice-Covered Seas, vol. 1, Univ. of Helsinki, Helsinki, pp. 105-194. 